\title{
Le criminologue et la victime
}

\section{Gianvittorio Pisapia}

\section{Introduction.}

En Italie, une sensibilité envers les victimes de crimes ne s'est pas encore développée. Même les rares initiatives mises en place n'ont pas enregistré de progrès significatifs dans l'identification d'une compétence opérationnelle. La tentation d'emprunter des raccourcis attrayants a pris le dessus en favorisant des interventions de type assistanciel suivant la phase de victimisation. Je ne veux pas sous-estimer l'importance de l'aide et du soutien à ceux qui ont été des victimes, mais on ne peut s'empêcher de manifester sa perplexité : ne s'occuper des victimes de crimes que dans la phase suivant l'événement, risque de devenir un alibi pour ne pas s'engager dans les dynamiques sociales qui portent de la condition de victime potentielle à celle de victime effective. Ce risque avait été considéré lors de la première initiative en faveur des victimes mise en place, en Italie, par une administration municipale (la Commune de Padoue). Je me réfère au Centro Iniziative Vittima (CIV) ${ }^{1}$ (Centre pour les Initiatives en faveur des Victimes) pour les victimes de la

\footnotetext{
- Professeur de «Criminologie », de «Sociologie de la déviance» et de "Criminologie et politique criminelle », Département de Philosophie, Sociologie, Pédagogie et Psychologie appliquée (FISPPA), Université de Padoue, Italie.

1 L'expérience du CIV s'est développée en trois phases. La phase préparatoire (octobre 1992-janvier
}

délinquance quotidienne, que l'on appelle erronément petite délinquance.

Cette expérience a fait comprendre qu'il est inutile de créer un centre d'aide aux victimes en cas d'absence de conditions minimales pour une alliance opérationnelle entre l'administration locale, les institutions préposées au contrôle social et le secteur privé et social. Cet enseignement a été toutefois ignoré par des initiatives successives qui se sont développées dans quelques régions italiennes.

Je reviens à la question qui avait accompagné la décision d'aborder ce problème : «Pourquoi s'occuper des victimes de crimes ?». La question perd son aspect rhétorique si l'on ne répond pas spontanément qu'il est juste de s'engager en faveur de la victime, puisque cette dernière ayant subi un préjudice, vit une situation de souffrance. Faut-il s'occuper de la victime parce que sa présence sociale indique des lacunes dans le système du contrôle social ? Où parce qu'elle met en évidence les limites des politiques sociales de ceux qui se sont engagés dans des activités de prévention et de réinsertion des personnes qui posent des problèmes sociaux ?

Répondre par l'affirmative à ces questions, conduit inévitablement à se demander si la victime est aujourd'hui encore, en Italie, la protagoniste absente (exception faite pour les initiatives adressées aux femmes victimes de violence conjugale et aux enfants abusés et 1993) ; la phase de démarrage (février 1993); la phase 
maltraités). Est-ce que cela signifie que l'on n'a pas dûment affronté le problème du contrôle, de la prévention et de la réinsertion sociale ? Est-ce que cela signifie qu'il y a une carence de solidarité ?

Il s'agit de questions auxquelles on ne peut guère échapper, étant donné qu'affronter la question de la victimisation dans une perspective criminologique, implique la nécessité d'affronter aussi ces aspects. Si je devais aujourd'hui me prononcer, en tant que criminologue, sur l'opportunité de mettre en œuvre des services d'aide aux victimes, je répondrais que des initiatives de ce genre devraient représenter non pas le point de départ, mais le point d'arrivée d'un parcours caractérisé par la prise en charge des responsabilités par les administrations locales, les institutions préposées au contrôle et le secteur privé et social. Un service spécialisé devrait être le reflet d'une politique sociale qui a déjà appliqué une logique de participation de la collectivité. Cette politique devrait considérer la victime non seulement comme un utilisateur, mais comme une ressource pour la construction d'une politique territoriale convaincante. Une ressource dont il serait naturellement souhaitable de se passer.

Les experts et les intervenants italiens, réussiront-ils à construire une compétence en ce domaine ? C'est un défi que les criminologues doivent relever. La criminologie a été traditionnellement indiquée comme la science qui s'occupe non seulement de faits délictueux, des auteurs de crimes, des processus de criminalisation, des différentes formes de

de conclusion (mars-juin 1993). réaction sociale vis-à-vis de la criminalité et de la déviance, mais aussi des victimes.

Toutefois, si l'on me posait la question : «Estimez-vous qu'il faudrait mettre en place, en Italie aussi, des centres qui s'occupent des victimes de crimes? », je serais très prudent si je répondais par l'affirmative. S'agit-il d'une prudence excessive ? C'est justement en m'occupant de victimes que je suis arrivé à la conviction qu'en Italie il faut déployer des efforts ultérieurs au niveau de la réflexion méthodologique.

La proposition de Padoue, l'hypothèse de départ, peut être résumée de la façon suivante.

a) Il aurait été stérile d'affronter les situations individuelles de victimisation (dont l'on n'entendait certainement pas sous-estimer l'importance) si l'on n'avait pas réussi à les relier aux problèmes concernant le contrôle social;

b) Par ailleurs, il aurait été également stérile de s'occuper de la question sociale si les interventions n'avaient pas été mises en œuvre en veillant tout particulièrement à la souffrance individuelle des victimes.

Allier la dimension individuelle à la dimension collective, exige un travail interprofessionnel et interinstitutionnel pour qu'un projet devienne un choix partagé avec des objectifs communs et des stratégies d'intervention différentiées, mais interactives.

Dans cette perspective, il est également nécessaire de réélaborer la conception de criminologie traditionnelle. Dans ces pages, la criminologie est proposée comme une recherche conceptuelle autant qu'opérationnelle des conditions pour sauvegarder la complémentarité 
et la mise en relation des apports de connaissances que les différentes disciplines et professions élaborent sur la criminalité, la conduite anti-juridique, la victimisation et le contrôle social, lorsque l'on a une confrontation interdisciplinaire et interprofessionnelle ${ }^{2}$.

\section{La compétence méthodologique du criminologue.}

Face aux multiples dimensions de la réalité du crime et de la victimisation, la constitution d'un savoir appelé «criminologie » ne prend un sens que si l'on suppose qu'un discours unitaire ne peut résulter d'une synthèse et d'une intégration des connaissances et des informations issues des différentes disciplines qui s'occupent de la question criminelle.

Pour qu'il y ait une interaction entre des disciplines et des professions caractérisées par des statuts, des règles et des méthodologies hétérogènes, il est nécessaire de prévoir un espace conceptuel commun, à l'intérieur duquel les questions et les réponses résultant d'une démarche commune prennent corps.

La criminologie traditionnelle se présente comme un domaine disciplinaire polycentrique et comme une discipline de synthèse des connaissances sur les auteurs de délits, les faits délictueux, le contrôle social, les situations antisociales et les victimes de délits produits par les différentes disciplines. C'est pourquoi, elle n'a pas réussi à trouver un dénominateur commun et a engendré des contradictions insurmontables.

\footnotetext{
${ }^{2}$ En ce qui concerne notre démarche théorique, on renvoie à nos textes : La dimensione normativa della criminologia, Cedam, Padova, 1992; Limes. Le criminologue en action, Monographic, Sierre, 1997; Manuale operativo di criminologia, Cedam, Padova, 2013.
}

C'est pourquoi, je pense qu'il est nécessaire de remettre en discussion l'approche de la criminologie traditionnelle et de passer à une criminologie qui soit avant tout en mesure d'offrir des descriptions et des explications de la question normative. J'entends par là l'ensemble des questions sociales et des politiques institutionnelles que l'on peut relever lorsqu'on se pose le problème de la rencontre et de l'affrontement avec les normes de conduite. A cela s'ajoutent les problèmes d'élaboration et de réélaboration des règles d'interaction sociale et des procédures, ainsi que ceux de la structuration et de la restructuration des modalités de comparaison avec les règles constitutives et reconstitutives du contexte.

Déjà en 1983, je m’étais demandé s’il était possible d'aborder les thématiques de la question criminelle sans avoir préalablement affronté les nœuds de la question réglementaire et la réponse que je suggérais était négative. Le besoin d'identifier une dimension originale de la criminologie avait fini par mettre au centre la question réglementaire. Dans sa dimension publique, celle-ci relève des normes, des règles et des procédures composant l'organisation d'une société, considérée comme un tissu de rapports entre les individus, de relations de réciprocité et d'interactions.

Sur le plan individuel, on se demandait s'il était raisonnable d'affronter la déviance des normes juridiques sans faire référence à l'expérience normative qu'un sujet acquiert au cours de sa $v^{2}{ }^{3}$. Une action anti-juridique fait toujours suite

\footnotetext{
3 Le terme «expérience » renvoie à la rencontre du sujet avec des personnes, des actes, des situations permettant l'acquisition de connaissances : l'expérience est l'observation et l'écoute de la réalité, l'occasion pour l'interroger, pour dévoiler ce qu'elle
} 
à des expériences de rencontre avec les normes, les règles et les contraintes présentes dans le contexte.

Une réflexion ultérieure nous avait rendu conscients du fait que l'idée de transgression ne pouvait être cristallisée dans la déviance des règles de conduite, notamment des règles juridiques. La signification originale $\mathrm{du}$ mot «transgresser» renvoie à l'idée de marcher, d'avancer et de dépasser ses propres limites pour pénétrer dans l'espace vital d'autrui. La transgression peut être positive lorsque deux ou plusieurs personnes décident, de commun accord, de partager leurs propres espaces. Elle devient négative lorsqu'une personne, hôte indésirable, envahit le territoire d'autrui, par exemple dans le cas de la victimisation, qui est le non-respect des limites de l'identité d'autrui.

\section{Le criminologue, opérateur de médiation.}

Si l'on examine les compétences des disciplines faisant partie des sciences humaines, on se rend compte qu'aucune d'entre elles n'est en mesure d'affronter les définitions sociales de phénomènes tels que les délinquants, la délinquance, les victimes des crimes et le contrôle social.

D'où l'exigence d'une confrontation entre les différentes disciplines et les professions qui touchent à la question criminelle. Chaque discipline interagissant avec les autres, produit

cache. Elle se caractérise par un parcours exploratoire de la complexité et par une construction de l'aspect problématique de la réalité. Le terme "normative», lié au terme expérience, indique que l'attention se porte sur les aspects de l'expérience qu'un sujet mûrit en se rapportant à des normes déjà codifiées, ou bien en contribuant à construire des règles et des procédures rendant possible la confrontation sociale. des fragments de connaissance. Si l'on veut éviter que ces fragments ne soient éparpillés à tout jamais sur une terre qui n'appartient à personne, il est raisonnable d'imaginer un espace qui s'étend le long des limites des différents territoires du savoir et à l'intérieur duquel ces fragments convergent et interagissent. L'on peut penser à une zone écotone $e^{4}$, qui est à la fois une terre du milieu et une terre de frontière. Cette zone qui marque une nette distinction entre deux ou plusieurs domaines du savoir, permet de les relier. Dans cette terre du milieu, convergent les fragments de connaissance et d'expérience qui interagissent lorsque l'on affronte des situations problématiques concernant le domaine des normes, des règles, des procédures et leur éventuelle transgression.

Cet espace contient les éléments typiques des secteurs disciplinaires et professionnels (droit, médecine légale, pédagogie, psychiatrie, psychologie, sociologie...) qui entrent en contact et conservent leur autonomie étant donné que les frontières ne sont pas effacées. C'est toujours dans cet espace que sont produites des connaissances originales supplémentaires par rapport à celles des aires adjacentes.

Par conséquent, la criminologie ne constitue plus, comme dans l'optique traditionnelle, le domaine disciplinaire dans lequel on fait la synthèse et on intègre les connaissances sur les

\footnotetext{
${ }^{4}$ On appelle " écotone » la transition écologique entre deux ou plusieurs systèmes différents ; par exemple, le passage de la forêt à la prairie, ou d'un substrat marin sablonneux à celui rocheux. Cette zone de frontière et de transition peut avoir une etendue linéaire considérable, mais toujours inférieure à celle des systèmes adjacents. Les systèmes écotones contiennent non seulement plusieurs organismes des systèmes de juxtaposition, mais aussi des organismes caractéristiques se limitant souvent à l'écotone et s'ajoutant à ceux des systèmes adjacents.
} 
auteurs de délits, les faits délictueux, la conduite anti-juridique, le contrôle social, les situations antisociales et les victimes des crimes, produites par le droit, la médecine, la psychiatrie, la psychologie et la sociologie.

Si l'on conçoit la criminologie comme un projet de connexion et d'interrelation, il en découle une image du criminologue, chercheur et opérateur, dont le but est d'élaborer des catégories d'analyse et des stratégies opérationnelles. Les disciplines et les professions traitant la question normative pourraient ainsi les adopter et en faire des ressources complémentaires en surmontant leurs limites.

Dans cette perspective, une des tâches du criminologue est de contribuer à créer les conditions (en termes d'hypothèse, finalité, objectif, stratégie, action, règles et procédures) pour que les processus d'interaction disciplinaire et professionnelle ne se limitent pas à un assemblage d'apports disciplinaires.

Il s'agit d'un effort considérable qui ne peut être limité à une activité de coordination délicate certes, mais essentiellement pratique. Il revient au criminologue de légitimer une compétence méthodologique, ce qui nécessite l'acquisition d'une méthode, c'est-à-dire des règles et des procédures avec lesquelles on peut atteindre le but qu'on s'est proposé. Il s'agit d'une compétence de connexion entre ces fragments de savoir qui permettent de dépasser les limites des différentes disciplines et professions, au moment où l'on active les interactions.

La méthode qui semble la plus fonctionnelle est celle de la médiation, comme modalité de régulation disciplinaire et professionnelle, concernant non seulement la phase qui suit immédiatement la confrontation, mais aussi les processus grâce auxquels nait et se développe l'interaction entre les disciplines et les professions.

Cette activité de médiation ne vise pas à éliminer les conflits éventuels (et parfois nécessaires) qui peuvent surgir entre les différentes parties composant le terrain de la question normative, mais à transformer tous conflits éventuels en opportunités de connaissance, en ressources opérationnelles et en occasions de découvertes pour de nouveaux canaux de communication.

Affirmer que le criminologue est la figure professionnelle de la connexion opérationnelle, c'est définir, de façon totalement conventionnelle, une potentialité conceptuelle. Celle-ci met en jeu les domaines d'étude (la délinquance, la victime de crime, le contrôle social...), les sujets (l’auteur du délit, le détenu, la victime de crime...) et les domaines opérationnels (la prison, le foyer pour jeunes délinquants, la famille, l'école...) qui relèvent des compétences de plusieurs disciplines et professions. Ce faisant, elle contribue à valoriser l'autonomie et la spécificité des différents apports en favorisant une comparaison critique et constructive.

Les criminologues traditionnels n'ont d'ailleurs jamais eu de compétence originale. Ils se sont occupés de la victime (en tant que victimologues), de l'auteur du délit (en tant que psychiatres ou psychologues), de la réaction sociale (en tant que sociologues), de la phase de détention (en tant que pénitentiaristes) ainsi que des tenants et aboutissants délictueux (en tant que juristes). Le criminologue aurait dû en seconde instance tenter une synthèse des différents apports. Au niveau opérationnel, le criminologue était donc destiné à assumer la 
dimension anthropologique de la psychiatrie et de la psychologie, le niveau expressif de l'action du droit ou de la sociologie, les finalités de la politique criminelle. Par conséquent, il était inévitable de constater le morcellement des différents aspects d'une situation sans aucune possibilité de les unifier.

\section{La victime : opérer en termes de situation.}

Dans notre optique, l'opérativité du criminologue trouve sa place à l'intérieur d'un parcours complémentaire entre les connaissances et les pratiques mises en œuvre par les disciplines et les professions traitant la question normative. De plus, elle fait partie d'une compétence méthodologique de médiation entre les instances disciplinaires et professionnelles.

Il s'agit d'une pratique qui se développe par une dynamique circulaire fondée sur la partie résultant de l'interaction. Cette pratique se traduit par la transformation des fragments de savoir qui échappent à la grille de lecture des autres disciplines dans des projets d'intervention. Cette potentialité projectuelle se conjugue difficilement avec la logique de l'intervention en situation de difficulté. Celle-ci prend en compte la réalité des sujets et des événements sur la base de l'évidence phénoménique, et retient une modalité opérationnelle fondée sur des compétences, des rôles et des tâches définis d'une façon rigide et standardisée.

Prenons le cas de la création d'un centre d'aide aux victimes d'actes criminels, mis en place sur proposition d'une administration locale. Le but $\mathrm{du}$ centre est de fournir une assistance juridique, économique et psychologique à ceux qui ont subi un crime. L'intervention du centre sera jugée satisfaisante si elle fournit des réponses, même partielles, à des situations individuelles sur la base de compétences disciplinaires et professionnelles faisant partie de l'activité. Un aspect fondamental du problème «victime de crime », celui du contrôle du territoire, n’est pas abordé, mais on offre des réponses en termes de services pour les besoins de ceux qui ont subi l'acte criminel.

On peut intervenir de cette façon dans la mesure où l'on estime que les conditions pour agir, par rapport à la complexité du problème, ne sont pas réunies ou lorsque l'administration locale n'est pas impliquée dans l'élaboration des politiques sociales. Ces dernières s'intéressant aux raisons et aux manières à cause desquelles on devient victime : un tel type d'intervention ne permet pas de cerner le processus de victimisation, en laissant de côté la question du contrôle du territoire.

Dans ce cas, la tâche du criminologue serait de vérifier la logique sur laquelle on base l'intervention et de faire émerger les contradictions qui découlent de la perspective opérationnelle de la situation. Le criminologue pourrait, par exemple, souligner la manière dont le fait de circonscrire l'activité au support individualisé risquerait d'avaliser la tendance de la victime de crime à reproduire un rôle passif par rapport aux autres problèmes sociaux. De façon cohérente, il devrait se charger de promouvoir l'élaboration d'un projet spécifique d'intervention qui partirait de la souffrance de la victime - aspect qu'on ne doit certainement pas sous-estimer - pour arriver aux situations complexes qui font de chaque citoyen une victime potentielle de conduites socialement négatives. 
Le criminologue devrait alors se poser certaines questions : quels aspects du problème seraient négligés si l'intervention n'était caractérisée qu'en termes de support (psychologique, d'assistance, légal, médical...) individualisé ? Quels aspects du problème seraient négligés si l'intervention, tout en tenant compte de la valeur relationnelle de chaque événement particulier de victimisation, ignorait les renvois institutionnels ? Autant d'interrogations qui pourraient se traduire ainsi sur le plan opérationnel : quelle aide effective offre-t-on à la victime si on se limite à envisager la dimension individuelle de l'expérience de victimisation en laissant inexplorée la réalité territoriale au sein de laquelle s'est produit le processus de victimisation ? Quelles réponses offre-t-on effectivement à la victime si on n'analyse pas les points-clés du contrôle de la criminalité, de l'utilité des activités visant à prévoir des situations socialement négatives, de l'efficacité des interventions ayant pour but de faire rentrer les auteurs des crimes dans la société libre?

\section{La victime : opérer en termes de problème.}

Opérer en termes de problème exige une logique d'intervention qui traduise les instances et les demandes de ceux qui ont été impliqués dans des situations socialement significatives par des actions répondant non seulement aux instances les plus immédiates, mais visant aussi à affronter leur signification sociale.

Prenons encore l'exemple d'une administration locale qui a décidé de créer un service de soutien psychologique aux victimes de crimes. Dans ce cas, il est plausible que cette initiative se traduise par une mise en place de consultations psychologiques.

En opérant dans cette perspective, que l'on pourrait définir une perspective d'assistance, il n'est pas prévu que l'on prenne en charge la tutelle des droits de la victime par rapport, par exemple, à la procédure judiciaire (avec référence aux modalités prévues pour porter plainte, aux délais prévus pour les témoignages dans la procédure pénale, aux démarches nécessaires à l'obtention d'un éventuel dédommagement...).

Le passage d'une intervention basée sur une logique d'assistance à une intervention ayant une approche que l'on pourrait définir de réparation et de tutelle, exige la présence de représentants d'organismes comme la magistrature et les forces de police. En leur absence, un organisme local ne serait pas en mesure d'agir sur les conséquences survenant après l'événement de victimisation.

Si une administration locale avait décidé de sauvegarder les intérêts du citoyen, par rapport à la procédure judiciaire aussi, elle devrait intervenir sur les droits de la victime, même si cela exige une demande de modification du système de justice. D’où la nécessité de se confronter à des secteurs institutionnels souvent réticents à accepter ce type d'ingérence.

Si une administration locale se proposait - sans ignorer pour autant les besoins individuels d'analyser le processus de victimisation, c'est-àdire d'examiner la façon dont tout citoyen peut passer de l'état de victime potentielle à celui de victime effective, elle serait obligée d'effectuer un progrès qualitatif ultérieur : elle devrait suivre une démarche opérationnelle que l'on peut appeler démarche communautaire et de régulation sociale. 
Dans cette approche communautaire et de régulation, on ne peut se limiter aux seuls besoins de la victime. Il devient nécessaire de développer des actions orientées vers l'efficacité du contrôle du territoire de la part des forces de l'ordre, le rôle des politiques préventives de l'organisme local, les effets du traitement pénitentiaire...

Par conséquent, pour ce qui est de l'intervention, il faudrait attribuer un rôle de premier plan non seulement aux victimes effectives, aux intervenants concernés par ce problème et aux représentants des organismes institutionnels, mais aussi aux victimes potentielles. Ce, pour élaborer des hypothèses, mettre en place des stratégies et définir des actions qui soient le patrimoine commun non seulement des professionnels du secteur, mais aussi des citoyens.

Quand on agit en termes de problème, la finalité n'est pas uniquement de donner des réponses pratiques à chaque situation, mais de définir aussi les modalités, les stratégies et les objectifs permettant de mettre l'accent sur l'importance sociale des aspects mis en évidence par les situations spécifiques. En d'autres termes, le but est de créer les conditions pour une expérience conceptuelle collective.

\section{La victime : projeter et programmer l'intervention.}

Si l'on choisit une logique d'intervention en termes de problème, il faut adopter une modalité opérationnelle de projet, c'est-à-dire une perspective qui puisse mettre en discussion, le cas échéant, les finalités de l'intervention préalablement définies ainsi que les modalités opérationnelles qui ne s'avéreraient fonctionnelles que pour la gestion des situations existantes.

Adopter une logique de projet, signifie encore une fois mettre en discussion des compétences considérées comme acquises. Pour atteindre cet objectif, il est cependant nécessaire de codifier les instruments de régulation des processus décisionnels, de programmer et de formaliser les procédures de travail. Cela, afin d'organiser la collaboration en évitant, de part et d'autre, l'acceptation de niveaux de médiation pouvant dénaturer la spécificité des tâches et des rôles, et réduire la possibilité de mettre en discussion, sans motif plausible, les accords sur la base desquels sont envisagées les interventions.

En outre, il est important de sauvegarder l'idée selon laquelle il est possible d'obtenir des résultats qui dépassent les initiatives spécifiques, sans quoi les interventions resteraient plus ou moins articulées - les unes par rapport aux autres -, tout en étant quand même susceptibles de se désarticuler dans la pratique opérationnelle quotidienne.

Les hypothèses de travail, les stratégies d'action, les formes d'intervention et les modalités de collaboration doivent ainsi être le résultat d'une concertation collective. Faute de quoi, il devient difficile, voire même impossible, de mettre en place des programmes d'intervention compréhensibles et auxquels tous puissent adhérer. C'est l'appropriation de cet espace qui permet de vérifier si l'on a sauvegardé les potentialités des compétences de tous ceux qui ont un rôle dans les situations, et si les interventions ont été mises en place et développées sans qu'il y ait eu une sélection arbitraire des éléments constitutifs des situations. 
Il est du ressort du criminologue de traduire la potentialité conceptuelle par des projets spécifiques. L'opérativité du criminologue prend, par conséquent, la forme d'un parcours d'exploration, ouvert à la nouveauté et à l'imprévu. Un parcours qui doit être, d'une part, suffisamment flexible pour s'adapter constamment à l'évolution des situations et, de l'autre, caractérisé par une logique de programme permettant au criminologue de réunir la spécificité d'exploration de l'intervention à une méthodologie claire et précise.

\section{Bibliographie.}

- Balloni A., sous la direction de, Vittime, crimine, difesa sociale, Clueb, Bologna, 1989.

- Balloni A., Bisi R., Sette R., Vittime e Vittimologia. Percorsi di studio e di ricerca, Minerva, Bologna, 2012.

- Bisi R., Sette R., "Victimes et Victimologie dans l'Italie d'aujourd'hui", in Les Cahiers de la sécurité, 2013, 23, pp. 142-151.

- Lopez G., Victimologie, Dalloz, Paris, 1997.

- Pisapia G.V., La vittima di reato tra controllo e sicurezza. Una riflessione operativa, Logos, Padova, 2014.

- Zauberman R., Robert Ph., Du côté des victimes: un autre regard sur la délinquance, L'Harmattan, Paris, 1995. 